\title{
PATHOLOGY
}

Gargin V.V., Kurchanova Yu.V., Ivanteeva Yu.I.

\section{THE POSSIBILITIES OF MUSEUM STUDYING OF VACTERL SYNDROME}

\author{
Kharkiv National Medical University, Ukraine
}

\begin{abstract}
The article deals with VACTERL association, particularly non randomly associated birth defects, typically defined by the presence of at least three of the following congenital malformations: vertebral anomalies, anal atresia, cardiac malformations, tracheo-esophageal fistula, renal anomalies, and limb abnormalities. Museum collection of the Department of Pathological Anatomy of Kharkiv National Medical University, which devoted to prenatal and perinatal pathology, has numerous museum exhibits representing this pathology. The specimens help to discuss pathological anatomy of each of these defects.
\end{abstract}

KeyWords: fetus, VACTERL, congenital defect.

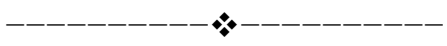

\section{INTRODUCTION}

VATER association was originally named in the early 1970 s with the description of seven patients as including at least three of the following features: Vertebral defects, Anal atresia, Tracheo-Esophageal fistula, Radial and Renal dysplasia [1]. Shortly thereafter, additional features, such as Cardiac malformations and additional Limb abnormalities, were added, and the condition was called VACTERL association. Nowadays VACTERL association (VA) is described as the non-random co-occurrence of vertebral defects (V), anal atresia (A), cardiac malformations (C), tracheo-esophageal fistula/esophageal atresia (TEF/EA), renal anomalies (R), limb abnormalities (L) [2]. VACTERL association is estimated to occur in approximately 1 in 10,000 to 1 in 40,000 live-born infants, depending on the exact criteria and the type of ascertainment used [2]. The works investigating clinical presentation of VACTERL patients and probing of aforementioned components are limited [3].

\section{Corresponding Author:}

Gargin Vitaly, MD, PhD, Professor, Pathological Anatomy Department, Kharkiv National Medical University, Ukraine. Email: vitgarg@ukr.net
Moreover, the studies in neonates and mature fetuses are restricted to clinical analysis [4] and morphological research as ultrasound investigation is carried out in termination of pregnancy with severe congenital abnormalities. At the same time such experience is important for the development of clinical thinking of future doctors.

Part of the museum collection of the Department of Pathological Anatomy of Kharkiv National Medical University devoted to prenatal and perinatal pathology is one of the most representative among the academic collections in the world. The majority of specimens in the exposition arrived much earlier than the wide use of the term VACTERL in diagnosing malformations especially in the post-Soviet countries.

It is necessary to take into account that it may determine incorrect interpretation as some cases may be wrongly diagnosed according to VACTERL description.

\section{PURPOSES, SUBJECTS and METHODS:}

\subsection{Purpose}

The aim of the present study is to assess the significance of the study of museum specimens allowing to trace VACTERL syndrome. 
accompanied by genitourinary (GU) anomalies including

\subsection{Subjects \& Methods}

The study implied literature search and the assessment of macroscopic preparations of the museum of Department of Pathological Anatomy at KhNMU dedicated to pre- and perinatal pathology with congenital malformations where VACTERL syndrome could be presented.

\section{Conflict of interests}

There is no conflict of interests.

\section{RESULTS AND DISCUSSION}

Thus, among patients with VACTERL $60-80 \%$ of individuals have vertebral abnormalities with a possible injury of any vertebra. This pathology may involve single or multiple vertebrae and may vary in severity. Characteristic vertebral anomalies include segmentation defects and may be accompanied by rib anomalies and abnormal spinal curvatures. In cohort of adult patients, approximately $25 \%$ of medically significant malformations have component features of VACTERL association, including $40 \%$ vertebral ones [5]. The museum specimens demonstrate multiple abnormal curvature of the spine, scoliosis, syncephalus and other types of spinal abnormalities. Thoracopagi always have various forms of vertebral anomalies, which are presented in Fig.1.
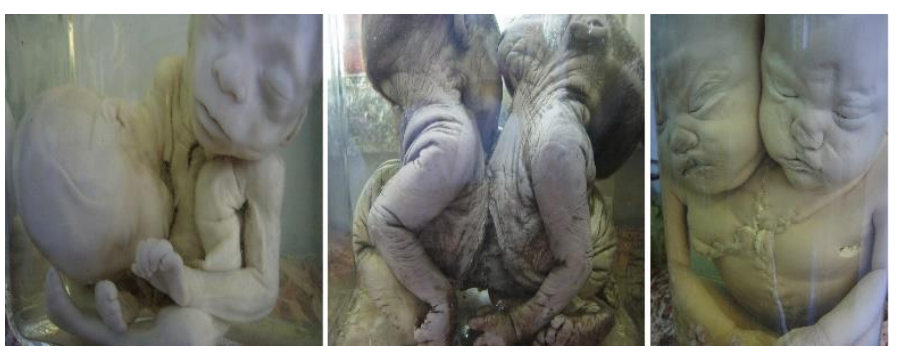

Fig.1 - Different types of thoracopagi

Anorectal malformations are reported in $60 \%$ of affected individuals with VACTERL [6] and are important in clear detection for surgical manipulation. Defects are frequently complex cloacal malformations. The museum collection of numerous congenital malformations is representative of such type of disorders.

Cardiac malformations have been reported in $70 \%$ of affected individuals. This pathology also includes vascular anomalies. Museum specimen of cardiac ectopy is a common example of this pathology. Ectopia cordis is a congenital malformation in which the heart is abnormally located either partially or totally outside of the thorax (Fig.2).
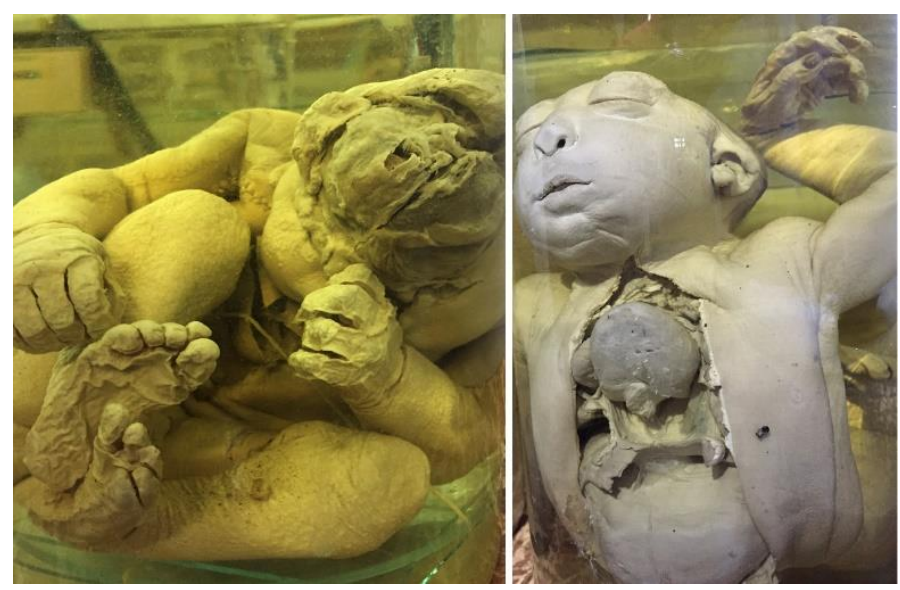

Fig.2 - Ectopia cordis associated with cerebral hernia (left) and anencephaly (right).

Tracheo-esophageal malformations are always characterized together because of their close anatomical location with common combination with Anorectal malformations. Tracheo-esophageal fistula (TEF) may be with or without esophageal atresia. A variety of TEF types have been described in $60 \%$ of individuals with VACTERL association, while esophageal atresia has been described in 70\%. [6]. Early interventions, including those related to fluid and nutrition management, can decrease complications (e.g., aspiration).

Renal anomalies, which may be accompanied by ureteral and GU anomalies, have been described in $70 \%$ of affected individuals [2]. The pathology is represented by an irregular shape of kidneys, absence of one or both organs. Optimizing adequate bladder emptying is especially important in obstructive hydronephrosis. 
Limb anomalies are the most visible form of congenital malformations in VACTERL association. It has the largest number of different manifestations [7-9]. They are represented by such malformations as amelia, sirenomelia, brachydactyly, syndactyly, monobrachia, hypodactyly, which occur in $40-50 \%$ of cases [7-9]. Amelia is the birth defect of lacking one or more limbs. It can also result in a shrunken or deformed limb. This pathology is shown in Fig.3.

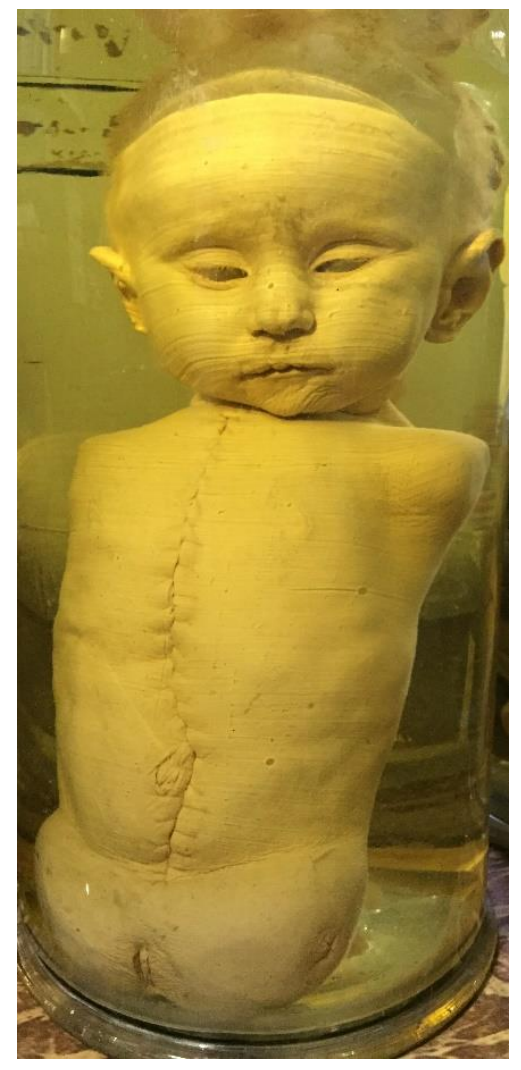

Fig.3 - Mature female amelia

Sirenomelia or «mermaid syndrome» is a very severe syndrome of caudal regression (complex malformation the caudal portion of the embryo), which is a rare severe congenital malformation of the distal spine and spinal cord [9]. Its clinical picture is accompanied by hypoplasia of the lower half of the trunk and extremities, a fusion of the lower limbs (fig.4).

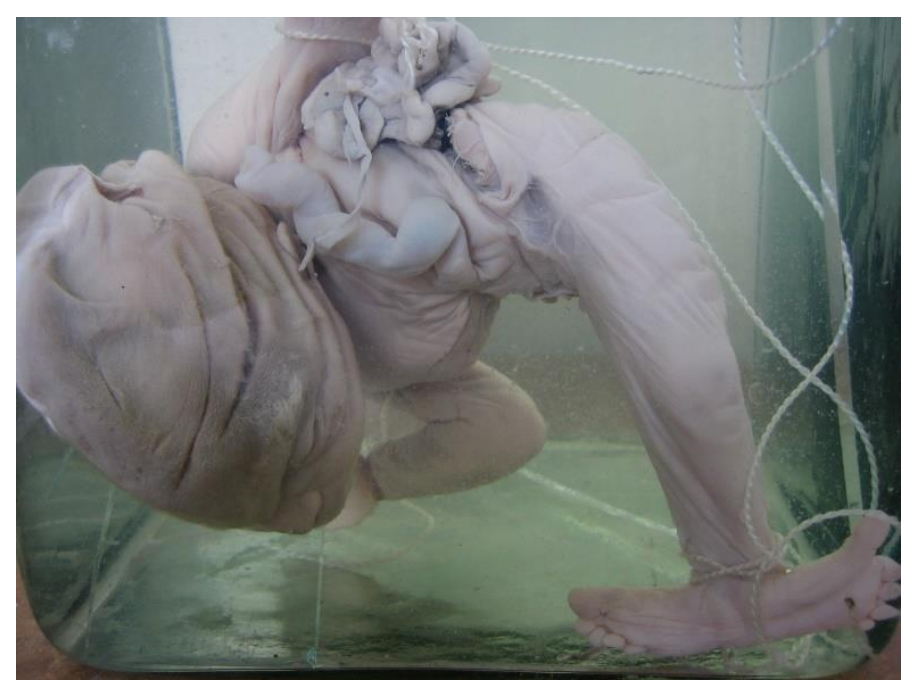

Fig. 4 - Antenatally died fetus with sirenomelia. (Born from a 16-year-old pregnant with addictive injections in history. She reported no familial history of congenital anomalies or diabetes)

The same applies regarding its relationship with narrow pelvis syndrome and VACTERL (vertebral defect, anal atresia, interauricular communication; interventricular communication, tracheal and esophageal atresia, and renal or radial agenesis) syndrome [10].

Mermaid syndrome is a very severe form of caudal regression syndrome (complex malformation of the caudal portion of the embryo), which, in turn, is a rare severe congenital malformation of the distal spine and spinal cord (its clinical picture is accompanied by hypoplasia of the lower half of the trunk and extremities, fusion of the lower limbs). Fusion can occur within the bone or soft tissues only. Presentation is characterized by renal agenesis, blindly ending colon, absence of external and internal genitalia, single umbilical artery atresia of the anus in most cases of sirenomelia $[9,11]$.

The etiology of the syndrome of caudal regression is not fully understood. Most authors name such causative factors as maternal diabetes, genetic predisposition, and insufficient blood supply to the lower half of the body of the fetus in the development of this pathology. In a normal embryo two umbilical arteries pump blood from the fetus 
to the placenta and one umbilical vein, which returns blood from the placenta to the fetus. Most infants with mermaid syndrome have only one umbilical artery and one vein. Rarely embryos with this syndrome develop typical two arteries and one vein $[9,12]$.

The study allowed us to select the following specimens to assess VACTERL presentation: amelia, sirenomelia, brachydactyly, syndactyly, monobrachia, hypodactyly, cardiac ectopia, many macroscopic preparations with multiple congenital malformations.

Inspection of the body allows to detect anal atresia and limb defects from seven features that are included in VACTERL syndrome for fetus or newborn. Anomalies of vertebrae, heart defects, tracheal abnormalities, esophageal atresia, renal anomalies could be detected without the study of the internal organs only with severe defects associated with injury of the integument, the presence of hernial protrusion.

These anomalies are formed during fetal development, caused by the interaction of multiple genetic and environmental factors [13-15]. Inheritance pattern is usually not observed, although there was a high frequency of disease manifestations in children born from mothers with diabetes. Involvement of genetic factors in the etiology of VATER/VACTERL association is suggested by various independent reports and studies in humans and animal models [15].

\section{CONCLUSIONS}

As we can see in the museum collection of the Department of Pathological Anatomy of Kharkiv National Medical University, VACTERL association can be observed in the museum specimens with multiple malformations. Based on the above we found numerous macroscopic specimens with VACTERL which can be used for teaching of future young specialists.

\section{REFERENCES}

1. Quan, L., Smith, DW. (1973). The VATER association. Vertebral defects, Anal atresia, T-E fistula with esophageal atresia, radial and renal dysplasia: a spectrum of associated defects. J Pediatr., 82, $104-107$.

2. Solomon, B.D. (2011) VACTERL/VATER association. Orphanet J Rare Dis, 6, 56.

3. Bartels, E., Schulz, A.C., Mora, N. W., PinedaAlvarez, D. E., Wijers, Ch. H. W., Marcelis, C. M.,... Reutter, H.M. (2012). VATER/VACTERL association: identification of seven new twin pairs, a systematic review of the literature, and a classical twin analysis. Clin Dysmorphol, 21(4), 191 - 195.

4. Salinas-Torres, V.M., Perez-Garcia, N., PerezGarcia, G. (2015) Clinical, Cytogenetic, Environmental and Inheritance Findings in Mexican Neonates with VACTERL Association. Indian J Pediatr, 82(1), 84-88

5. Raam, M.S., Pineda-Alvarez, D.E., Hadley, D.W., Solomon, B.D. (2011) Long-term outcomes of adults with features of VACTERL association. Eur J Med Genet., 54(1), $34-41$.

6. Lautz, T.B., Mandelia, A., Radhakrishnan, J. (2015) VACTERL associations in children undergoing surgery for esophageal atresia and anorectal malformations: Implications for pediatric surgeons. J Pediatr Surg, 50(8), 1245-1250.

7. Pelluard-Nehme, F., Baudet, Ch., Carles, D., Alberti, E. M., Delrue, M. A., Lacombe, D. (2007). A new case of VACTERL association with unilateral amelia of upper limb. Clinical Dysmorphology, 16,185 - 187.

8. Rougemont, A.-L., Bouron-Dal Soglio, D., Desilets, V., Jovanovic, M., Perreault, G., Oligny, L. L., Fournet, J. Ch. (2008). Caudal Dysgenesis, Sirenomelia, and Situs Inversus Totalis: A Primitive Defect in Blastogenesis. American Journal of Medical Genetics, 146A, 1470 - 1476.

9. Nikolaenko, E. U., Gargin, V. V. (2015). Sirenomelia as example of limb malformation. Inter collegas, 3, 317 - 322.

10. Fadhlaoui, A., Khrouf M., Gaigi, S., Zhioua F., Chaker A. (2010) The sirenomelia sequence: a case history. Clin Med Insights Case Rep, 3, 41-49.

11. Sepulveda, W., Martinez, J.L., Moenne, K. (2005) Sirenomelia (symelia dipus). Pediatr Radiol, 35(9), 931- 
933.

12. Tayel, S.M., Fawzia, M.M., Al-Naqeeb, N.A., Gouda, S., Al Awadi, S.A., Naguib, K.K. (2005) A morphoetiological description of congenital limb anomalies. Ann Saudi Med, 25(3), 219-227.

13. Chen, Ch.-P., Chang, T.-Y., Chen, Y.-Y., Chern, S.R., Su, J.-W., Wang, W. (2013). VACTERL association with hydrocephalus in a fetus conceived by in vitro fertilization and embryo transfer. Taiwanese Journal of Obstetrics \& Gynecology, 52, 575 - 579.

14. Solomon, B. D., Bear, K. A., Kimonis, V., de Klein, A., Scott, D. A., Shaw-Smith, Ch., Giampietro, Ph. F. (2012). Clinical Geneticists' Views of VACTERL/VATER Association. Am J Med Genet, 158A(12), 3087 - 3100.

15. Reutter H, Ludwig M. (2013) VATER/VACTERL Association: Evidence for the Role of Genetic Factors. Mol Syndromol, 4(1-2), 16-19.

Received: $\quad$ 02-Oct. - 2016

Accepted: 18-Dec. - 2016 